\section{Chemoprävention bei latenter Tuberkulose verbessern}

Menzies D et al. Four Months of Rifampin or Nine Months of Isoniazid for Latent Tuberculosis in Adults. N Engl ] Med 2018; 379: 440 - 453

Verschiedene Regime werden zur Chemoprävention der Aktivierung einer latenten Tuberkuloseinfektion (LTBI) eingesetzt. Ein gut etablierter Standard ist die 9-monatige Therapie mit Isoniazid. Die Adhärenzraten sind dabei allerdings gering, die Verträglichkeit ist nicht immer gut. Eine offene, randomisierte Studie verglich die Therapie mit Isoniazid mit der 4monatigen Behandlung mit Rifampicin, einer ebenfalls etablierten Chemoprävention bei LTBI.

Die Studie von Dick Menzies von der McGill-Universität in Montreal et al. wurde in neun Ländern weltweit durchgeführt. Randomisiert erhielten Erwachsene mit LTBI die 4-monatige Rifampicin-Therapie $(10 \mathrm{mg} / \mathrm{kg}$ täglich, maximal $600 \mathrm{mg}, \mathrm{n}=3.443)$ oder die 9-monatige Isoniazid-Theapie $(5 \mathrm{mg} / \mathrm{kg}$ täglich, maximal $300 \mathrm{mg}, \mathrm{n}=3.416$ ) ergänzt um Vitamin B6 bei denjenigen mit einem Neuropathierisiko. Die Autoren betonen, dass es bei dieser Studie keinen Sponsor gab, der teilweise oder ganz die Studienmedikation finanzierte. Sie untersuchte primär die Nichtunterlegenheit der Rifampicin-Therapie gegenüber der Isoniazid-Chemoprophylaxe hinsichtlich der Prävention einer aktiven TB innerhalb von 28 Monaten nach Randomisierung. Alle Ergebnisse wurden von einem unabhängigen Gutachtergremium beurteilt.

\section{Ergebnisse}

Eine bestätigte aktive Tuberkulose (TB) entwickelten in der Rifampicin-Gruppe über eine Beobachtungszeit von 7.732 Personenjahren 4 Patienten, ebenso viele klinische TB-Diagnosen wurden registriert. In der Isoniazid-Gruppe gab es bei einer Beobachtungszeit von 7.652 Personenjahren 4 bestätigte und 5 klinische Diagnosen. Die Differenz der Diagnoseraten lag unter 0,01 Fälle pro 100
Personenjahren sowohl für die bestätigte aktive TB (95\% Konfidenzintervall [KI] $-0,14-0,16)$ wie auch für eine klinisch diagnostizierte TB (95\% KI-0,23-0,22). Damit wurde die vorab definierte Nichtunterlegenheitsschwelle nicht überschritten. Eine Überlegenheit der 4monatigen Rifampicin-Therapie gegenüber der 9-monatigen Isoniazoid-Behandlung wurde nicht festgestellt. Es zeigte sich aber ein Unterschied in der Rate der vollständig abgeschlossenen Therapie von 15,1 Prozentpunkten (95\% KI 12,7-17,4). Auch war die Rate unerwünschter Ereignisse des Grads 3 - 5 innerhalb von 146 Tagen (120\% des 4-Monats-Zeitraums) um 1,1 Prozentpunkte geringer ( $95 \% \mathrm{KI}-1,9$ bis $-0,4)$. Der Unterschied in der Hepatotoxizität des Grads 3-5 lag bei 1,2 Prozentpunkten zugunsten von Rifampicin (95\% KI -1,7 bis $-0,7)$.

FAZIT

Eine Einschränkung der Studie ist neben dem offenen Studiendesign die niedrige Ereignisrate, die nicht geeignet ist, einen Unterschied aufzudecken, wie die Autoren zugeben. Die Studie bestätigt aber erneut die Vorteile einer verkürzten Chemoprävention mit Rifampicin mit einer höheren Rate komplett abgeschlossener Therapien und weniger Toxizität als mit Isoniazid bei gleich guter Effektivität. Damit stärkt die Studie die gute Evidenz für die Rifampicin-Chemoprävention laut der Autoren weiter.

Friederike Klein, München 